\title{
High-throughput screening and rational design to drive discovery in molecular water oxidation
} catalysis

\author{
Michael John Craig a and Max García-Melchor ${ }^{\mathrm{a}}{ }^{\mathrm{a}}$ \\ ${ }^{a}$ School of Chemistry, CRANN and AMBER Research Centres, Trinity College Dublin, College \\ Green, Dublin 2, Ireland.
}

*Correspondence should be addressed to: garciamm@tcd.ie

KEYWORDS: Water oxidation, oxygen evolution, density functional theory, high-throughput screening, reaction descriptors, scaling relations, catalyst design. 


\begin{abstract}
The difficulty of the oxygen evolution reaction is a fundamental impediment to the sustainable production of hydrogen, wherein molecular catalysts show the most impressive activity in terms of turnover frequency for this reaction. In this work, we have interrogated 444 automatically generated molecular water oxidation catalysts composed of well-known ligand scaffolds and six different transition metals ( $\mathrm{Cr}, \mathrm{Mn}, \mathrm{Fe}, \mathrm{Ru}, \mathrm{Co}$, and $\mathrm{Ni})$. This data confirms the method-independent universal scaling relationship for water oxidation catalysts, describes routes toward circumventing this relationship, and justifies the ascendency of Ru catalysts for this reaction. Leveraging this information while applying catalyst design principles, we summarize experimental results, giving credence to our prediction of 9 earth-abundant molecular catalysts with theoretical overpotentials ranging from 200 to $400 \mathrm{mV}$ as promising leads for experimental investigation. We also establish insights into spin-dependent scaling relations for key OER intermediates. Altogether, this work outlines the first steps towards enabling inverse design for molecular OER catalysts.
\end{abstract}




\section{Introduction}

The transition toward sustainable energy would be greatly facilitated by developing feasible means to store intermittent energy. ${ }^{1}$ One promising strategy is the production of hydrogen from renewably-driven water electrolysis, with the ability to use $\mathrm{H}_{2}$ as an energy-dense fuel to decarbonize sectors of industry and transportation. Aside from the merits of using it as a fuel, hydrogen is essential to fertilizer production, and finds industrial applications from oil refineries to electronics. ${ }^{2}$ However, this hydrogen is predominantly made via steam reforming of methane, contributing significantly to $\mathrm{CO}_{2}$ emissions. ${ }^{3}$

To date, the anodic oxygen evolution reaction (OER) is the principal challenge preventing electrochemical hydrogen production at scale. Cheap, efficient and performant catalysts for the OER would impel green $\mathrm{H}_{2}$ production and improve $\mathrm{CO}_{2}$ electroreduction, which also occurs at the cathode concurrently with the anodic reaction shown in Eq. 1.,5

$$
2 \mathrm{H}_{2} \mathrm{O}_{(\mathrm{l})} \rightleftharpoons \mathrm{O}_{2(\mathrm{~g})}+4 \mathrm{H}^{+}+4 \mathrm{e}^{-} \quad E^{0}=1.23 \mathrm{~V} \text { vs. RHE }
$$

To evolve oxygen, a variety of mechanisms are possible, including electron and proton transfer steps, which can be sequential or proton-coupled electron transfers (PCETs). The most commonly studied mechanisms are the water nucleophilic attack (WNA) mechanism and the interaction of two metal-oxo units (I2M), as depicted in Figure 1. 


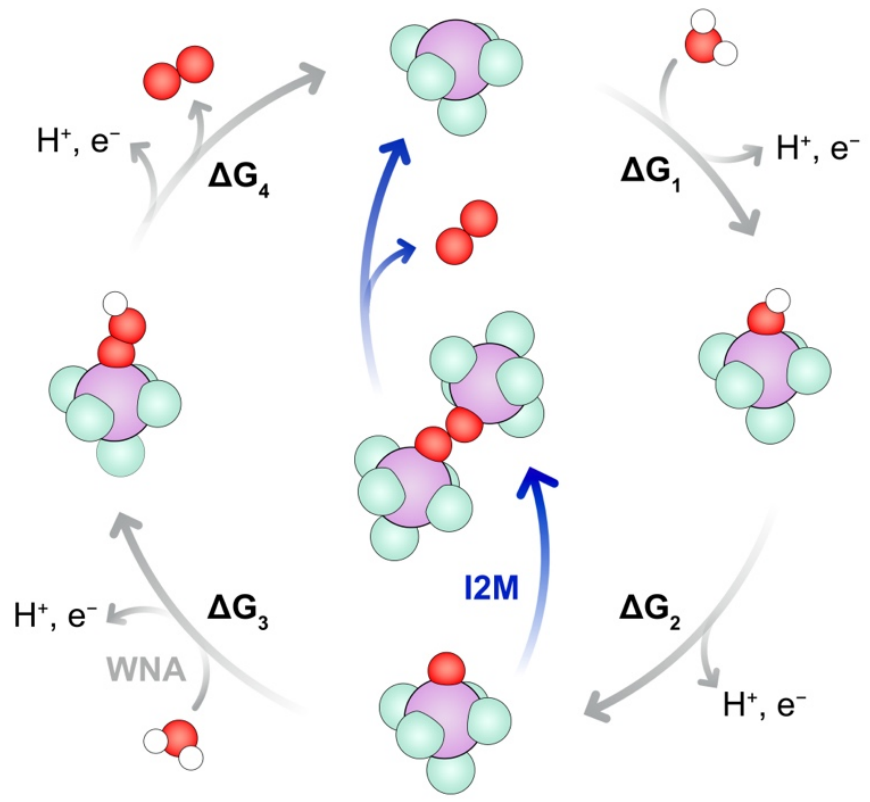

Figure 1. Catalytic cycle depicting the OER mechanisms via water nucleophillic attack (WNA) and the interaction of two metal-oxo units (I2M). Red, white and purple spheres represent oxygen, hydrogen and metal atoms, respectively and the teal spheres represent a general ligand skeleton.

Computational studies assuming the WNA mechanism typically describe Gibbs energy changes associated with each step in the WNA as functions of intermediate binding energies e.g. $\Delta G_{H O^{*}}$, as outlined in Eqs $2-5 .^{6}$

$$
\begin{aligned}
& H_{2} O+* \rightarrow H O^{*}+H^{+}+e^{-} \\
& \Delta G_{1}=\Delta G_{H O^{*}}-e U+k_{B} T \ln a_{H^{+}} \\
& H O^{*} \rightarrow O^{*}+H^{+}+e^{-} \\
& \Delta G_{2}=\Delta G_{O^{*}}-\Delta G_{H O^{*}}-e U+k_{B} T \ln a_{H^{+}} \\
& O^{*}+H_{2} O \rightarrow H O O^{*}+H^{+}+e^{-} \\
& \Delta G_{3}=\Delta G_{H O O^{*}}-\Delta G_{O^{*}}-G_{H_{2} O}-e U+k_{B} T \ln a_{H^{+}} \\
& H O O^{*} \rightarrow O_{2}+H^{+}+e^{-} \\
& \Delta G_{4}=\Delta G_{O_{2}}-\Delta G_{H O O^{*}}-e U+k_{B} T \ln a_{H^{+}}=4.92 \mathrm{eV}-\Delta G_{H O O^{*}}-e U+k_{B} T \ln a_{H^{+}}
\end{aligned}
$$


Where $U$ denotes the applied potential, $a_{H^{+}}$the activity of the protons, and the Gibbs energy of the overall reaction has been replaced by $4.92 \mathrm{eV}$ to address the well-documented issues with modelling the triplet ground state of the $\mathrm{O}_{2}$ molecule. ${ }^{7}$ These binding energies allow for the calculation of the theoretical overpotential, $\eta_{\text {theor }}$, according to Eq. 6.

$$
\eta_{\text {theor }}=\left\{\max \left(\Delta G_{1-4}\right) / e\right\}-1.23 \mathrm{~V}
$$

Linear scaling relationships have been established between the binding energies of the HO* and $\mathrm{HOO}^{*}$ intermediates using density functional theory (DFT) for heterogeneous and homogeneous OER catalysts, ${ }^{8-14}$ and can thereby be said to be universal. The essential consequence of these relations is that $\Delta G_{\mathrm{HO}^{*}}$ and $\Delta G_{\mathrm{HOO}^{*}}$ cannot be varied independently, so when they are not thermodynamically ideal, i.e. $\Delta G_{H O O^{*}}=\Delta G_{H O^{*}}+2.46$, they place a fundamental restriction on catalytic activity, as any catalyst which follows the conventional WNA mechanism will have a lower limit placed on the possible overpotential, a constraint often referred to as the "overpotential wall".

Recently, we highlighted the importance of a one-electron oxidation occurring at the metaloxo unit of certain molecular complexes, prior to $\mathrm{O}-\mathrm{O}$ bond formation, which must be considered to correctly describe the impressive activity of the best homogeneous OER catalysts. ${ }^{10}$ Furthermore, we showed that this additional step allows for the circumvention of the restriction imposed by the above scaling relation, leading to the possibility for catalysts to exhibit near-zero overpotentials provided the energy levels of redox intermediates are appropriately distributed. The elementary steps involved in this process and their associated Gibbs energy change are shown in Eqs. 7 and 8. The oxidation state of the oxo intermediates is given in parenthesis, and each adsorbate binding energy, $\Delta G_{x *}$, is defined in the Supplementary Note 1. 


$$
\begin{aligned}
& \mathrm{O}(\mathrm{IV})^{*} \rightleftharpoons \mathrm{O}(\mathrm{V})^{*}+\mathrm{e}^{-} \\
& \Delta G_{3 \prime}=\Delta G_{O(\mathrm{~V})^{*}}-\Delta G_{O(I V)^{*}}-e U \\
& \mathrm{O}(\mathrm{V})^{*}+\mathrm{H}_{2} \mathrm{O} \rightleftharpoons \mathrm{HOO}^{*}+\mathrm{H}^{+} \\
& \Delta G_{4 \prime}=\Delta G_{H O O^{*}}-\Delta G_{O(V)^{*}}+k_{B} T \ln a_{H^{+}}
\end{aligned}
$$

With this insight, we proposed a series of catalyst design principles to allow for the discovery of catalysts based on earth-abundant metals which can stabilize high oxidation states such as $\mathrm{Mn}$ and $\mathrm{Fe}$. Mononuclear first-row transition metal complexes based on $\mathrm{Cr},{ }^{15} \mathrm{Fe},{ }^{16-18} \mathrm{Mn},{ }^{19}$ $\mathrm{Co},{ }^{20-23} \mathrm{Ni},{ }^{24}$ and $\mathrm{Cu}^{25,26}$ have been shown to be active towards the OER. Despite these exciting developments, earth-abundant catalysts have yet to show comparable activity to those based on single-site Ru in terms of turnover frequency (TOF) and overpotential. ${ }^{27-29}$

In this study, we have evaluated and refined our design principles by studying intermediates across metals, ligands and oxidation states. Traditional computational OER studies apply the conventional OER descriptor, $\Delta G_{O^{*}}-\Delta G_{H O^{*}}$ which is thought optimal when it is equal to half the value of the intercept found in the scaling relationship between $\Delta G_{H O^{*}}$ and $\Delta G_{H O O^{*}}$. While this is effectively a $\mathrm{pH}$-independent descriptor, OER catalyst performance is known to be $\mathrm{pH}$ dependent, with activity varying significantly in alkaline or acidic conditions. This is especially relevant due to the exigency of developing catalysts which are active in acid. ${ }^{30}$ Alterations in activity due to $\mathrm{pH}$ can be attributed to interwoven factors such as catalyst instability, ionic transport, switching mechanisms or a combination thereof. In this study, we examine distinct reaction mechanisms by modelling descriptors in differing oxidation states, which is typically not done in studies utilizing the OER descriptor. Since one can expect more hydrogenated intermediates in acidic conditions, one would expect the extra oxidation mechanism to be less favored than in base. A concrete example of this is provided in an experimental Pourbaix 
diagram reproduced in Supplementary Figure 1. We believe such a simple consideration could allow for $\mathrm{pH}$-dependence using simple descriptors.

To test our design principles, herein we report a high-throughput computational screening of mononuclear complexes with the aim of accelerating the discovery of earth-abundant OER catalysts by focusing on non-critical elements which can stabilize high-valent $M(V)$ intermediates and deepening our fundamental understanding of this reaction. In particular, we present a fully-automated approach to create transition metal complexes with molSimplify ${ }^{31}$ via permutation of a selected set of ligands which have been previously seen in experimental OER catalysts. ${ }^{32}$ As shown in Figure 2, five different geometries are generated from these ligands depending on their denticity, each of them containing one of six metal centers (i.e. $\mathrm{Cr}$, $\mathrm{Mn}, \mathrm{Fe}, \mathrm{Ru}, \mathrm{Co}$, and $\mathrm{Ni}$ ), leading to a total of 444 molecular catalysts which are octahedral upon the addition of the adsorbate. For each catalyst, $\Delta G_{H O(I I I)^{*}}$ and $\Delta G_{H O O(I I I)}$ are evaluated, with $\Delta G_{H O(I V)^{*}}, \Delta G_{H O O(I V)^{*}}, \Delta G_{O(I V)^{*}}$ and $\Delta G_{O(V)^{*}}$ calculated for a subset of these complexes. 


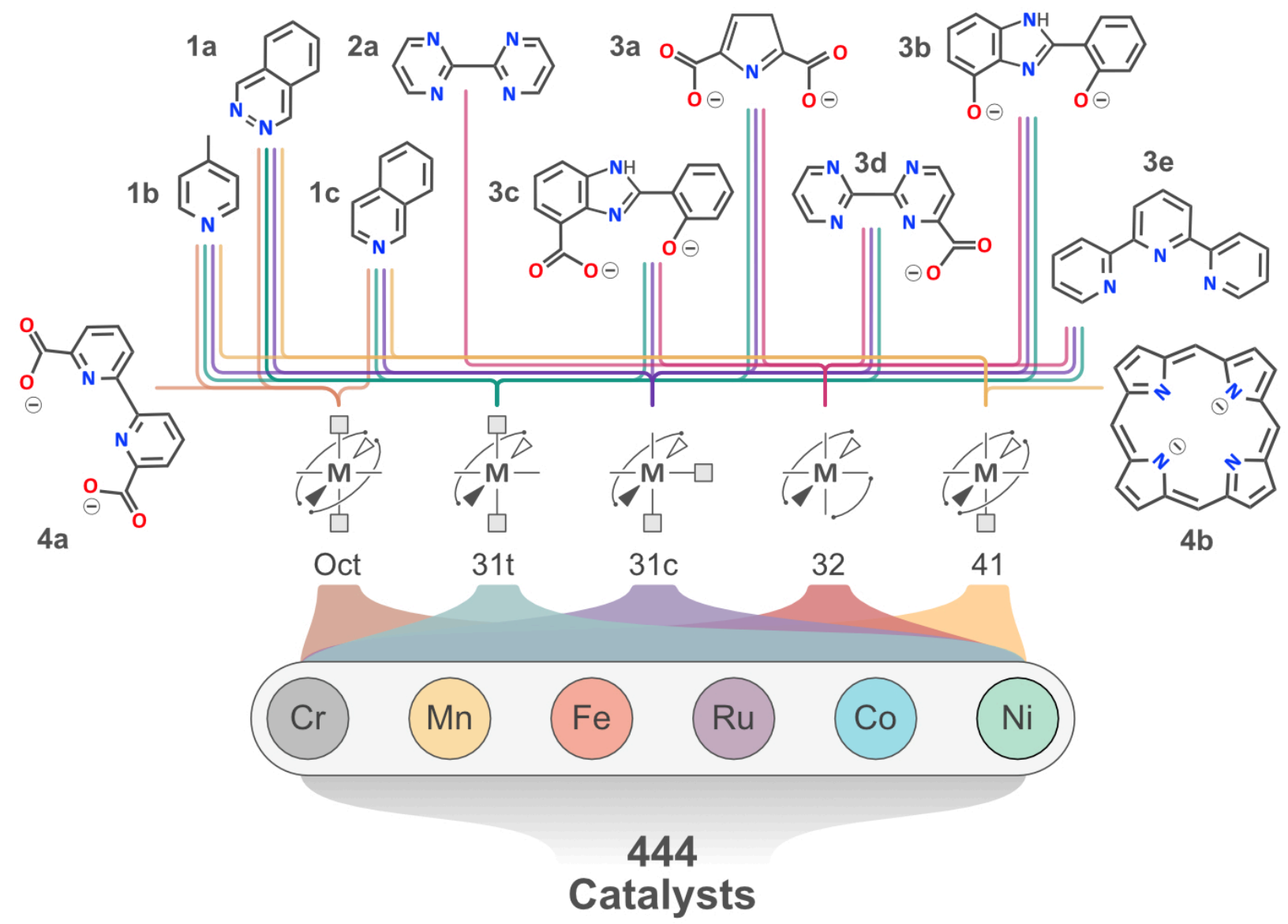

Figure 2. Depiction of the combination of ligands, geometries and transition metals used to automatically generate the OER complexes investigated in this work, with the label for each distinct geometry. Monodentate ligands in each of the geometries are represented by squares, while the adsorbate is placed at the end of the free lines protruding from the metal. Where there are two monodentate ligands, they can either be cis or trans to each other, leading to labels 31c or $31 \mathrm{t}$, respectively. The dynamic nature of the coordination environment in this catalyst has been previously studied by Govindarajan et al..$^{33}$

Our computational investigations probe the existence of universal, functional-independent OER scaling relations and re-examine fundamental assumptions of scaling relations between the $\mathrm{HO}^{*}$ and $\mathrm{O}^{*}$ intermediates. Our data also provides justifications for intriguing deviations from prototypical scaling relations that are a result of the spin states observed in the modelled open-shell systems. In addition, our automated high-throughput screening leads to 9 earth- 
abundant catalysts based on $\mathrm{Cr}$, Mn and Fe exhibiting a predicted OER overpotential lower than $400 \mathrm{mV}$. In all, our high-throughput analyses provide new insights on experimental findings and open new avenues for future research towards the discovery of low cost and efficient OER catalysts.

\section{Results and Discussion}

\section{Insights from Molecular OER Scaling Relations}

With the 444 catalysts formed via the automatic structure generation outlined in Figure 2, we modeled the $\mathrm{HO}(\mathrm{III}) *$ and $\mathrm{HOO}(\mathrm{III}) *$ intermediates to investigate the universality and methoddependence of the OER scaling relation between $\Delta G_{H O^{*}}$ and $\Delta G_{H O O^{*}}$. To confirm the robust nature of this relationship, we employed a set of four different density functional theory (DFT) methods, which include two widely used hybrid meta-generalized gradient approximation (mGGA) functionals exhibiting varying degrees of Hartree-Fock exchange, i.e. TPSSh${ }^{34}(10 \%)$ and $\mathrm{B} 3 \mathrm{LYP}^{35}(20 \%)$, as well as the m-GGA functional M06- $\mathrm{L}^{36}$ and the pure GGA functional BEEF-vdW. ${ }^{37}$ The latter functional also allows for the non-self-consistent evaluation of an ensemble of 2,000 separately parametrized GGA functionals, which permits the estimation of errors. ${ }^{37}$ This further demonstrates the functional-independent nature of the OER scaling relation by showing the results over a vast range of GGAs. Details outlining the application of these functionals is provided in the Computational Methods section. 
a)

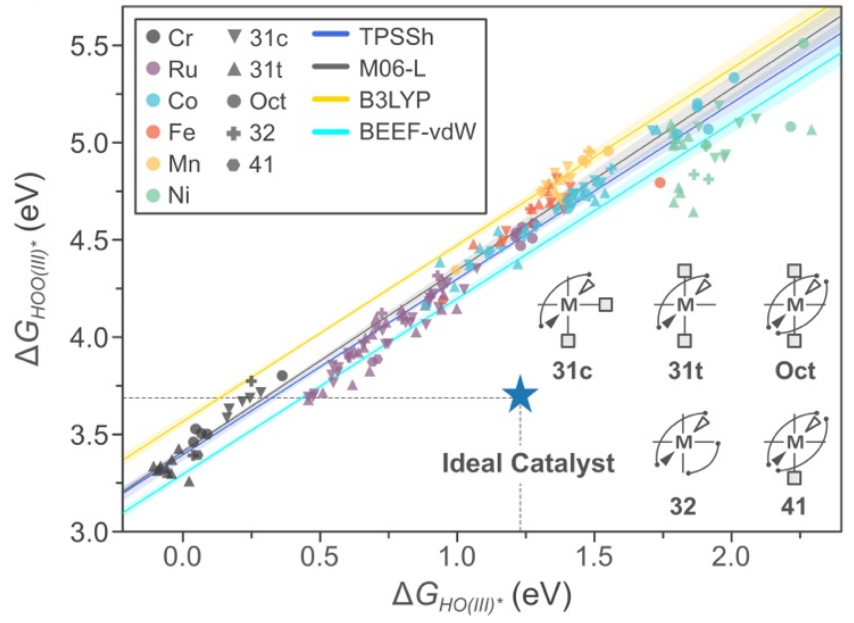

b)

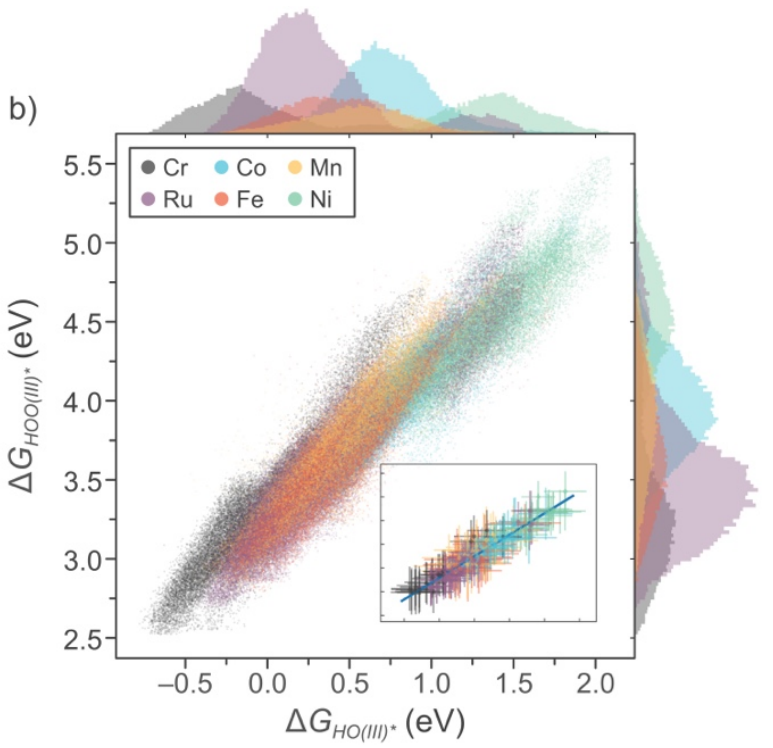

Figure 3. (a) Plot showing the OER scaling relationship between $\Delta G_{H O(I I I)^{*}}$ and $\Delta G_{H O O(I I I)^{*}}$ spanning different DFT methods with lines of best fit for the multiple methods and geometries. Data points correspond to the calculated TPSSh data. The ideal catalyst binding energies are indicated with the blue star. Each marker represents a distinct geometry as depicted in Figure 2, with each color representing a distinct metal. (b) Plot showing the OER scaling relationship across the model space of BEEF-vdW GGA functionals. This plot includes ensemble binding energies which exhibited a distance from the mean less than the standard deviation of the ensemble provided by BEEF-vdW. The distributions of individual values from these ensembles for $\Delta G_{H O(I I I)^{*}}$ and $\Delta G_{H O O(I I I)^{*}}$ are shown in the secondary axes to illustrate the funtionalindependent nature of the scaling relation using the BEEF ensemble. The inset shows the datapoints and associated errorbars of the BEEF-vdW data, along with the line of best fit through those points.

In Figure 3a, the results of TPSSh calculations for individual catalysts are shown as data points, while the lines of best fit through each functional are also provided, showing each line to be essentially parallel, with the intercept of the lines being the only appreciable difference. Notably, we find that this data can be used to begin to rationalize past decades of experimental 
research into monometallic OER catalysts..$^{32} \mathrm{Ru}$ complexes exhibit a tendency to shift below the line of best fit, meaning these complexes stabilize the HOO* intermediate to a greater extent than other metals, on average. This phenomenon, which reduces the minimum theoretical overpotential via the conventional WNA mechanism, was also noted in a computational study of $\mathrm{IrO}_{2}$ and $\mathrm{SrIrO}_{3}$ surfaces, and formed part of the rationalization for these materials' impressive activity in acidic media. ${ }^{38}$ We note that this effect is seen for each of the investigated DFT functionals to varying degrees, as displayed in the Supplementary Figure 2. Similar to $\mathrm{Ru}, \mathrm{Ni}$ catalysts are found to deviate from the line of best fit, which we will rationalize later.

Figure $3 \mathrm{a}$ also reveals that octahedral $\mathrm{Ru}$ complexes, shown as purple circles, exhibit a $\Delta G_{H O(I I I)}$ close to the ideal value of $1.23 \mathrm{eV}$, leading to a $\Delta G_{H O O(I I I)^{*}}$ of $4.5 \mathrm{eV}$. Notably, this particular family of Ru catalysts with the $\mathbf{4 a}$ ligand are some of the best molecular catalysts known to date. ${ }^{27,28}$ Through direct observations from Figure 3a, we posit that this is because they are not constrained to follow the conventional WNA pathway. Instead, these catalysts are known to undergo an extra oxidation step before forming the $\mathrm{O}-\mathrm{O}$ bond via the $\mathrm{I} 2 \mathrm{M}$ mechanism, ${ }^{27,28}$ and we recently showed that both facets must be considered to explain their favorable activity. ${ }^{10}$ These complexes, therefore, circumvent the scaling relationship by sidestepping the $\mathrm{HOO}^{*}$ intermediate.

To further confirm the robustness and method-independence of the OER scaling, we applied the error estimation framework utility from BEEF-vdW to represent that relation for GGA functionals which are within one standard deviation of the mean value of this ensemble (Figure 3b). This was performed similarly in a study on the functional-dependence of scaling relations, ${ }^{39}$ although to the best of our knowledge, this is the first time that the analysis in Figure $3 b$ has been done for the OER. In the GGA space, we find that the distribution of the 
binding energies for each metal is the same, which reinforces the functional independence of the OER scaling relation.

Interestingly, Figure 3a also indicates that the $\Delta G_{H O O(I I I)} *$ values for Ni(III) complexes deviate from the linear scaling relationship. We posit that this is because the $\mathrm{HOO}(\mathrm{III})^{*}$ intermediate allows for these complexes to be stabilized in the Ni(II) oxidation state, while the HO(III)* intermediate cannot. To support this, we inspected the Ni spin densities in $\mathrm{HOO}(\mathrm{III})^{*}$ and $\mathrm{HO}(\mathrm{III})^{*}$ intermediates to use them as a proxy for oxidation state, finding values of $c a .1 .5$ and 0.9 , respectively. This makes a further connection to recent experimental findings, wherein it has been shown that orienting a magnet appropriately to electrocatalysts composed of mixed $\mathrm{Ni}, \mathrm{Fe}$ and $\mathrm{Zn}$ oxides can significantly enhance the rate of the OER. ${ }^{40}$ The authors proposed that this effect stems from the induced magnetic field which facilitates the parallel spin alignment along the OER to yield the triplet oxygen product. Furthermore, a recent perspective has proposed that the magnetic enhancement effect could be related to a deviation from scaling relationships. ${ }^{41}$

This prompted us to investigate this hypothesis by inspecting the spin density in the HOO* intermediate and the deviation of the $\Delta G_{H O O(I I I)^{*}}$ values from the scaling shown in Figure 3 a. To do this, we describe the collinearity of oxygen atoms, $\lambda$, using the Mulliken spin densities, $\mu$, of the individual oxygens in the $\mathrm{HOO}^{*}$ intermediate as:

$$
\lambda=\mu_{O_{1}} \mu_{O_{2}}
$$

The result of plotting $\lambda$ along with the corresponding Mulliken spin density on the metal $\left(\mu_{M}\right)$ in the HOO* intermediate is shown in Figure 4, where we identify a distinct region defined by $\lambda>0.1$ and $\mu_{M}>1$ in which Ni catalysts deviating favorably from the OER scaling lie. 


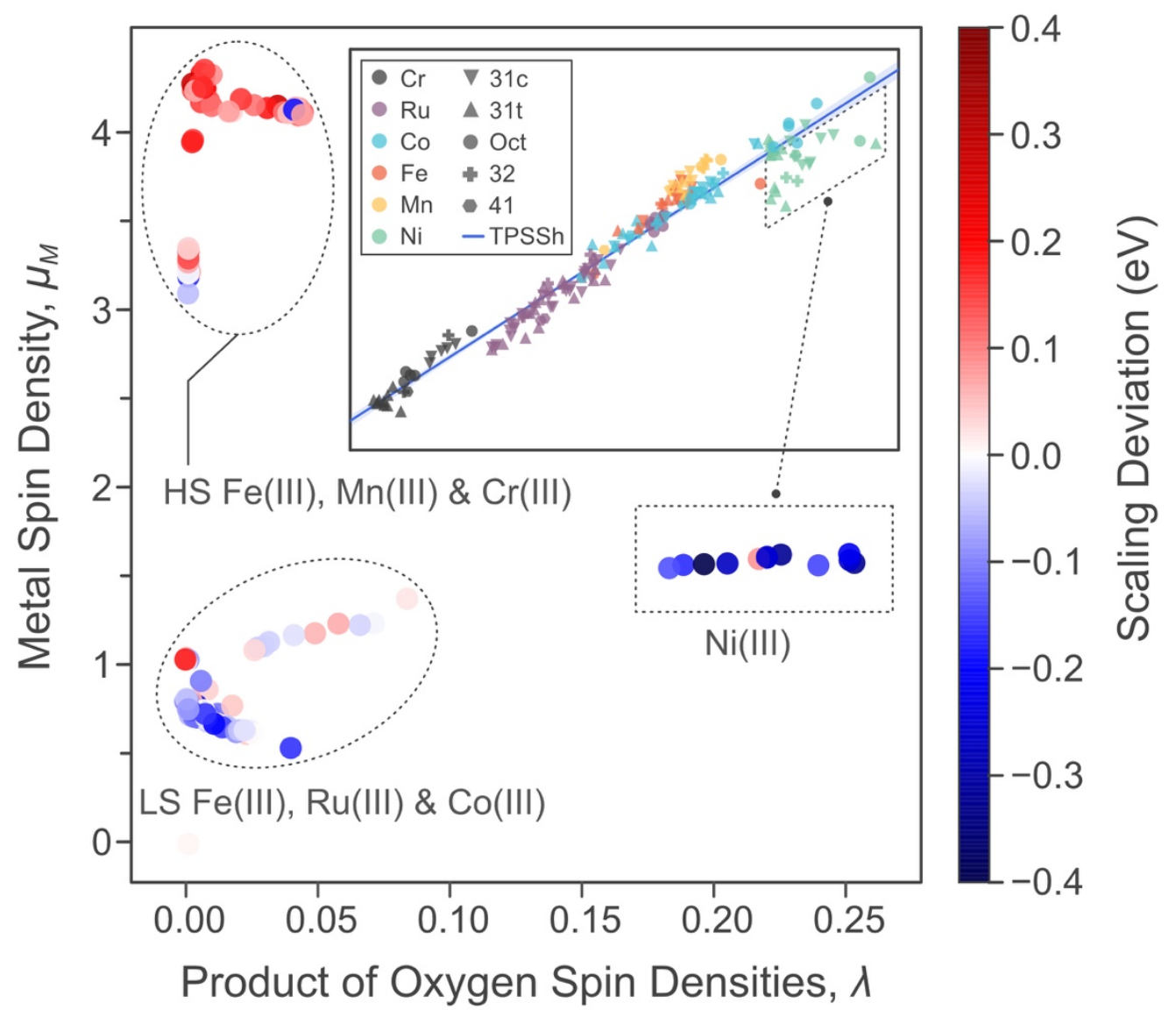

Figure 4. Graphical representation of the $\lambda$ parameter against the metal spin density, $\mu_{M}$, to rationalize the scaling deviation of the $\mathrm{HOO}^{*}$ intermediate for Ni catalysts. The colorbar represents the deviation from the TPSSh line of best fit from Figure 3a (inset). The rectangle and parallelogram are used to illustrate the essence of the relationship found. We note there is not a one-to-one correspondence between the data points in each shape, that is, not all points in the rectangle are in the parallelogram.

The remaining catalysts in Figure 4 are grouped into two regions. The upper left region denotes Fe(III), Mn(III) and Cr(III) complexes with high-spin (HS) $\mathrm{d}^{5}, \mathrm{~d}^{4}$ and $\mathrm{d}^{3}$ electronic configurations, respectively. These catalysts tend to lie above the line of best fit, with the exception of an Oct-Fe complex which shows a seven-coordinated HOO* intermediate. This geometry has been shown to be important in Ru OER catalysts. ${ }^{42}$ On the other hand, in the lower left region, we find $\mathrm{Fe}(\mathrm{III}), \mathrm{Ru}(\mathrm{III})$ and $\mathrm{Co}(\mathrm{III})$ catalysts with low-spin (LS) $\mathrm{d}^{5}, \mathrm{~d}^{5}$ and $\mathrm{d}^{6}$ 
configurations, respectively, which tend to lie below or close to the scaling line. Additionally, our calculations indicate that the ligand scaffold is not responsible for the deviation from the scaling, as H-bonding does not lead to the same decisive effect, as depicted in Supplementary Figure 3. Hence, we conclude that the magnetic enhancement effect can also be leveraged in the WNA mechanism to facilitate $\mathrm{O}-\mathrm{O}$ bond formation via the stabilization of the $\mathrm{HOO}^{*}$ intermediate relative to the established scaling.

The theoretical basis for spin-dependent oxygen electrochemistry has been developed and examined by Gracia and co-workers. ${ }^{43-45}$ In those works, this effect was claimed to be exchange-mediated, which is borne out by our analysis as we only observe this effect when using DFT functionals which include Hartree-Fock exchange, as shown in Supplementary Figure 4. Our contribution effectively cements the importance of this approach by revealing the connection between spin conservation and circumventing well-established scaling relations, which we envisage to be of paramount importance in identifying cost-effective OER materials.

\section{Rational Screening of Molecular Catalysts}

We next sought to test the possibility of near-zero overpotential catalysts via an extra-oxidation mechanism. ${ }^{10}$ With this aim, we computed the conventional OER descriptor, $\Delta G_{O(I V)^{*}}-$ $\Delta G_{H O(I I I)^{*}}$, for catalysts in Figure 3a which exhibit a value of $\Delta G_{H O(I I I)^{*}} \leq 1.5 \mathrm{eV}$, leading to the volcano plot presented in Figure 5a. Use of this volcano representation has found useful application across OER research. ${ }^{46,47}$ This upper limit was chosen to place a lower limit on the overpotential due to $\Delta G_{H O(I I)^{*}}$, where our aim is to find catalysts with overpotentials that circumvent the "overpotential wall". 

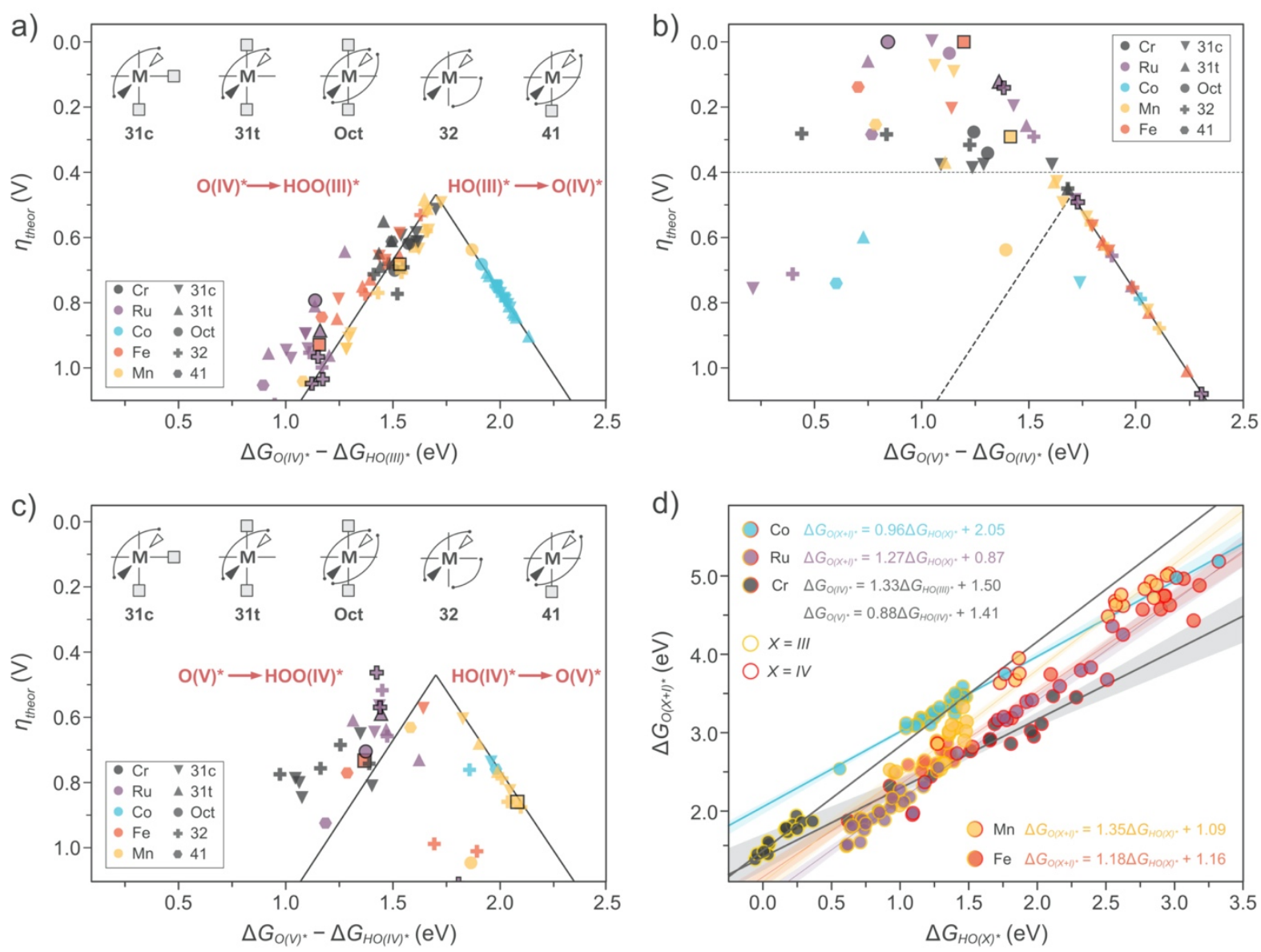

Figure 5. Two-dimensional volcano plots using (a) the conventional OER descriptor representing a PCET between oxidation states $\mathrm{M}(\mathrm{III})$ and $\mathrm{M}(\mathrm{IV})$, (b) the extra one-electron oxidation descriptor, in $\Delta G_{O(V)^{*}}-\Delta G_{O(I V)^{*}}$, with dashed line denoting the cutoff point of 400 $\mathrm{mV}$ overpotential and (c) the OER descriptor representing a PCET between oxidation states $\mathrm{M}(\mathrm{IV})$ and $\mathrm{M}(\mathrm{V})$. In a) and c) we present the presumed limiting step based on the scaling relations. The points with a black outline denote experimentally known OER catalysts, where we use a square to represent catalysts with pentadentate ligands, since this geometry is not represented in the set of catalysts generated as in Figure 2. d) Metal-specific, cross-oxidation state scaling relations with lines of best fit for each set of metal-specific relations. Outlines of points denote the oxidation state of the $\mathrm{HO}^{*}, \mathrm{O}^{*}$ pair. Two scaling relations are shown for $\mathrm{Cr}$ as the oxidation-state specific scaling relations differ considerably, which is not the case for the other metals. The shaded region represents the $95 \%$ confidence interval of the learned linear 
model, which is not included in the case of the $\mathrm{Cr}(\mathrm{IV})$ relation due to the large span of this interval, shown in Supplementary Figure 9.

While catalysts which are close to the peak of the volcano in Figure 5a demand a considerable overpotential, they evolve oxygen via PCETs exclusively, thereby avoiding the building up of positive charge and fostering redox potential levelling. ${ }^{48}$ The best leads for future experiments which exhibit a PCET-only mechanism are chosen so that $\eta<0.55$, (since this is close to the lower limit of $0.5 \mathrm{~V}$ imposed by the scaling in Figure $3 \mathrm{a}) \Delta G_{O(I V)^{*}}<\Delta G_{H O(I V)^{*}}$, and $\Delta G_{H O O(I I I)^{*}}<\Delta G_{O(V)^{*}}$, since we do not want to perform the extra oxidation in this case. This identifies a 32-Fe catalyst composed of the 3a tridentate and 2a bidentate ligands as seen in the labelled catalysts from Figure 2 . The 32-Fe catalyst is particularly interesting as we expect that the bipyrimidine ligand (labelled $\mathbf{2 a}$ in Figure 2) could be used to immobilize the catalyst onto a solid support. ${ }^{49}$ Also included in this plot as black-outlined datapoints are catalysts which are experimentally known to catalyze the OER.

We note that the volcano peak at $(1.7 \mathrm{eV}, 0.47 \mathrm{~V})$ in Figure $5 \mathrm{a}$ is larger than the overpotential wall previously reported by our group, which we attribute to the fact that individual metals have slightly different scaling relationships and intercepts, as seen in Supplementary Figure 5. This difference leads directly to a difference in overpotential walls. In a recent work, we reported a scaling relationship with an intercept of $3.26 \mathrm{eV}$, where the dataset of molecular catalysts contained $9 \mathrm{Ru}$ catalysts out of a set of 17 , which led to the scaling relation being overly representative of complexes with this metal.

We next investigated whether the complexes with $\Delta G_{O(I V)^{*}}-\Delta G_{H O(I I I)^{*}}<1.5 \mathrm{eV}$ can undergo the extra oxidation prior to $\mathrm{O}-\mathrm{O}$ bond formation by inspecting the one-electron oxidation descriptor $\Delta G_{O(V)^{*}}-\Delta G_{O(I V)^{*}}$, leading to the volcano plot shown in Figure 5b. This step is reasoned to be crucial in finding low-overpotential earth-abundant catalysts for this reaction, 16 
and we note that it is required to justify the activity of the Fe and Mn mononuclear catalysts of the same geometry as the set of catalysts examined in this study. In particular, we correctly predict near-zero overpotential at $\mathrm{pH}=0$ for an amidate-ligated iron complex via the extraoxidation mechanism, conferring with the experimental Pourbaix diagram which showed that this step occurred at $c a .1 .25 \mathrm{~V},{ }^{50}$ while correctly describing the Mn complex with an anionic $\mathrm{N}$-donor ligand as low overpotential $(<0.4 \mathrm{~V})$, and within the redox potential of the chemical oxidant used. ${ }^{19}$ This agreement with experiment is not possible without considering the extra oxidation, which affords greater confidence in our predictions of the OER activity. Also noteworthy is the fact that multiple earth-abundant catalysts lie within the region of low overpotential with $\eta<0.4 \mathrm{~V}$, although we detail how to further filter catalysts before ultimately suggesting promising leads.

Implicit in the extra-oxidation mechanism is the assumption that the one-electron transfer occurs immediately prior to the formation of what we assume to be the site for the water nucleophilic attack, the $\mathrm{M}(\mathrm{V})-\mathrm{O}$ intermediate. However, this $\mathrm{M}(\mathrm{IV})-\mathrm{O}$ to $\mathrm{M}(\mathrm{V})-\mathrm{O}$ oxidation could occur at any point before this, leading to the M(IV)-OH intermediate, which would be subject to the same constraints on the overpotential inherent in the scaling relation in Figure 3, as evidenced in the oxidation-state independent scaling in Supplementary Figure 6. As a result, the OER descriptor can be applied in the same way at a higher oxidation state, although because pairs of $\mathrm{HO}(\mathrm{III}) / \mathrm{HO}(\mathrm{IV})$ and $\mathrm{O}(\mathrm{IV}) / \mathrm{O}(\mathrm{V})$ binding energies are uncorrelated, the OER descriptors at varying oxidation states are also uncorrelated, as shown in Supplementary Figure 7. This stresses the importance of sampling the correct oxidation states when using this descriptor.

The volcano plot for this higher oxidation OER descriptor is shown in Figure 5c. Interestingly, we find that $\mathrm{Ru}$ and $\mathrm{Cr}$ catalysts predominate in the left leg of the volcano, implying a relative 
ease in the formation of the $\mathrm{M}(\mathrm{V})$ oxidation state, while Fe candidates lie on either side, and Mn and Co exclusively lying to the right leg of the volcano. In calculating the overpotential for Fig. $5 \mathrm{c}$, we calculated the Gibbs energy changes associated with going between the most stable intermediate for a given oxidation state, an approach which our group has applied to molecular OER catalysts with two active sites. ${ }^{51}$ Note that in doing this, there are points which deviate significantly from the faces of the volcano, since the reaction intermediate we are considering does not necessarily determine the overpotential.

Taking the data presented in Figures 5a-c, we establish metal-specific scaling relations between $\Delta G_{H O(I I I, I V)^{*}}$ and $\Delta G_{O(I V, V)^{*}}$, shown in Figure 5d. Notably, Co and Cr exhibit different slopes to the set of $\mathrm{Mn}, \mathrm{Fe}$ and $\mathrm{Ru}$ catalysts, while two scaling relations are shown for $\mathrm{Cr}$ as the linear fit for oxidation-state specific scaling relations differ considerably for $\mathrm{Cr}$, which is not the case for the other metals. Another interesting facet of these linear fits is the difference in the intercepts of the $\mathrm{Mn}, \mathrm{Fe}$ and $\mathrm{Ru}$ set of metals, wherein the oxo-formation energy of $\mathrm{Ru}$ at a given binding energy, for either oxidation state, is $c a .0 .2 \mathrm{eV}$ lower than for Fe and $\mathrm{Mn}$ for either oxidation state, thus indicating that forming high-valent intermediates is comparatively easier for $\mathrm{Ru}$ in complexes with the same $\Delta G_{H O(I I I, I V)^{*}}$.

The sensitivity of the slope to separating by metal in the scaling relations requires further discussion. On the basis of the d-band model, ${ }^{52}$ which is the bedrock of scaling relations, these slopes should equal 2. However, there are often deviations from this behavior in studies of both heterogeneous and homogeneous catalysts. ${ }^{14,53}$ The seminal justification for predicting a slope of 2 was predicated upon assumptions derived from effective medium theory that less electron density is required to stabilize metal active sites upon the addition of a hydrogen atom to an $\mathrm{O}$ adsorbate. ${ }^{54}$ Yet, we note that this would not be the case in radical metal-oxyl $(\mathrm{M}-\mathrm{O} \bullet)$ fragments since the electron which forms the bond with $\mathrm{H}$ would not have been associated to 
the electron density of the complex, but only with the adsorbate. In fact, we observe this for Co, wherein the spin density on the metal centre remains the same in going from $\mathrm{HO}$ to $\mathrm{O}$ despite the d-electron count varying from $d^{4}$ to $d^{6}$. This is enabled by the oxyl moiety (along with the organic ligand at $\mathrm{Co}(\mathrm{V})-\mathrm{O}^{*}$ and $\left.\mathrm{Co}(\mathrm{IV})-\mathrm{OH}\right)$ hosting spin density to allow for the reduction of cobalt to a LS-Co(III) with a d ${ }^{6}$ electronic configuration. For this reason, the bonds between the $\mathrm{Co}-\mathrm{O}(\mathrm{IV}, \mathrm{V}) *$ and $\mathrm{Co}(\mathrm{III}, \mathrm{IV})-\mathrm{HO}^{*}$ species are of similar order (Supplementary Table S1), leading to a slope of $c a .1$ in the scaling plot depicted in Figure 5d. A similar effect is observed in $\mathrm{Mn}(\mathrm{IV})-\mathrm{O}, \mathrm{Fe}(\mathrm{IV}, \mathrm{V})-\mathrm{O}, \mathrm{Ru}(\mathrm{IV}, \mathrm{V})-\mathrm{O}$, with the metal only slightly oxidised in going from $\mathrm{HO}^{*}$ to $\mathrm{O}^{*}$ complexes. From these observations, we can approximate the expected slope for these metals as:

$$
m_{\text {pred. }(\uparrow \uparrow)}=2-\frac{\Delta(\operatorname{spin} \text { on } O)_{H O^{*} \rightarrow O^{*}}}{\Delta(\operatorname{spin} \text { on } M-O)_{H O^{*} \rightarrow O^{*}}}
$$

where the numerator denotes the average spin density change (for a given metal) occurring on the oxygen in the adsorbate in going from the $\mathrm{HO}^{*}$ to $\mathrm{O}^{*}$ intermediate, and the denominator denotes the average total spin density change in both the metal and the bound oxygen. Using Eq. 10, we note a remarkable agreement between the observed slopes in $\mathrm{Ru}(\mathrm{IV}, \mathrm{V})-\mathrm{O}, \mathrm{Fe}(\mathrm{IV}$, V)-O and Mn(IV)-O which further confer with the general trend from Wiberg bond orders ${ }^{55}$ for the $\mathrm{M}-\mathrm{O}$ and $\mathrm{M}-\mathrm{OH}$ intermediates, seen in Supplementary Table 1. However, we note that this equation falters in the case of $\mathrm{Mn}$ and $\mathrm{Cr}$ as the metal-oxygen spin pairing is antiferromagnetic in nature for $\mathrm{Cr}(\mathrm{V})-\mathrm{O}$ and a mix of both ferromagnetic and antiferromagnetic for $\mathrm{Mn}(\mathrm{V})-\mathrm{O}$ species. Indeed, spin-based non-linear d-band model refinements have been proposed for ammonia adsorption on heterogeneous catalysts. ${ }^{56}$ Creating lines of best fit through the antiferromagnetic complexes in Supplementary Figure 7, we observe a slope less than 1 . In these cases, the slope can be predicted by using Eq. 11 . 


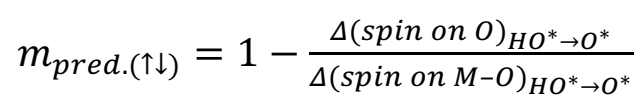

The results of using Eq. 11 to predict the slopes of lines of best in Figure 5d are also outlined in Table 1, wherein we see an impressive agreement in predicting the slopes for $\mathrm{Mn}(\mathrm{V})-\mathrm{O}$ and $\mathrm{Cr}(\mathrm{V})-\mathrm{O}$. We can now see that the slope of the combined $\mathrm{Mn}(\mathrm{IV}, \mathrm{V})-\mathrm{O}$ scaling relation is the combination of the ferromagnetically and antiferromagnetically coupled species, which leads to different slopes when separately fitted. Finally, while there is an interesting predictive ability in using these equations, we note that $\mathrm{Cr}(\mathrm{IV})-\mathrm{O}$ intermediates behave as $\mathrm{Cr}$-oxo species, which can also be confirmed from the calculated Wiberg bond orders in Supplementary Table 1. As such, one would expect them to exhibit a slope of 2 since this is the expected value for precisely this type of bond, based on the bond order conservation principle, which our equations do not predict well. However, we note that the cluster of $\mathrm{Cr}(\mathrm{IV})-\mathrm{O}$ points (black circles with yellow outlines in Figure 5d) are the most closely-packed subset of data, which may obscure the true scaling relation due to the sensitivity of the lines of best fit to new points that lie outside the bounds of the considered points.

Table 1. Predicted slopes using the spin-based descriptors from Eqs. 10 and 11. The slope $m$ was found by fitting the line of best fit to the relevant subset.

\begin{tabular}{lccc}
\hline Catalyst Set & $\boldsymbol{m}$ & $\boldsymbol{m}_{\text {pred.( } \uparrow)}$ & $\boldsymbol{m}_{\text {pred.( } \uparrow \downarrow)}$ \\
\hline $\mathrm{Co}(\mathrm{IV}, \mathrm{V})$ & 0.96 & 1.06 & - \\
$\mathrm{Ru}(\mathrm{IV}, \mathrm{V})$ & 1.27 & 1.25 & - \\
$\mathrm{Fe}(\mathrm{IV}, \mathrm{V})$ & 1.18 & 1.24 & - \\
$\mathrm{Mn}(\mathrm{IV}, \mathrm{V})^{\mathrm{a}}$ & 1.66 & 1.43 & - \\
$\mathrm{Mn}(\mathrm{V})^{\mathrm{b}}$ & 0.92 & - & 0.76 \\
$\mathrm{Cr}(\mathrm{IV})$ & 1.33 & 1.96 & 0.96 \\
$\mathrm{Cr}(\mathrm{V})$ & 0.88 & - & 0.85 \\
\hline
\end{tabular}


a Slope fitted by including only $\mathrm{Mn}(\mathrm{V})$ species which are ferromagnetically spin-coupled, of which there were four, shown in Supplementary Figure 8.

${ }^{\mathrm{b}} \mathrm{Mn}(\mathrm{V})-\mathrm{O}$ species with antiferromagnetic spin coupling, seen in Supplementary Figure 8.

Hence, it is clear that altering adsorbates does not affect all metal active sites in a uniform way, an implicit assumption of cross-metal scaling relations. These results highlight the value of studying the effect of fitting subset-specific scaling relations and make a compelling case for revisiting scaling relations involving $\mathrm{O}^{*}$ and $\mathrm{HO}^{*}$ binding energies and using magnetism to guide deviations away from these relations towards optimum activity.

We note that the Co data in Figure 5d also agrees with our recent studies of Co-based polyoxometalates (POMs), which found that these catalysts cannot show overpotentials less than $0.8 \mathrm{~V}$ due to the restriction placed on oxygen evolution by the $\Delta G_{H O(X)^{*}}$ vs. $\Delta G_{O(X+I)^{*}}$ scaling relation, ${ }^{14}$ while also predicting that all Co catalysts will always have $\Delta G_{O(I V, V)^{*}}-$ $\Delta G_{H O(I I I, I V)^{*}}>\Delta G_{H O O(I I I, I V)^{*}}-\Delta G_{O(I V, V)^{*}}$. This implies that Co-based molecular catalysts have poor activity and that they cannot benefit from the extra oxidation step. These findings stress the need for future reports of Co-based homogeneous catalysts to rule out the presence of Co-oxide nanoparticles which can form in solution, giving the false impression of molecular catalytic activity. ${ }^{57}$

With all the knowledge acquired above and using the data from Figure 5b, we can now propose catalysts for experimental realization on the basis of predicted overpotentials less than $400 \mathrm{mV}$ and consisting of earth-abundant metals. For this, we filter candidates by enforcing that $\Delta G_{O(V)^{*}}<\Delta G_{H O O(I I)^{*}}$ so the extra oxidation is possible, and that the Gibbs energy change associated with the atom-proton transfer (APT), $\Delta G_{H O O(I I I)^{*}}-\Delta G_{O(V)^{*}}$, be less than $1 \mathrm{eV}$, as this is the largest value exhibited by the set of transition metal complexes known experimentally to catalyze the OER (in particular, the red square in Figure 5b). This leads to 9, 
predominantly Cr-based, low overpotential catalysts, shown in Figure 6. While the low overpotentials in these catalysts are very encouraging, we also need to consider the conditions under which the extra oxidation mechanism is expected occur. In particular, to properly determine which mechanism is expected to occur, the $\mathrm{p} K_{a}$ for the $\mathrm{HO}(\mathrm{IV})^{*} / \mathrm{O}(\mathrm{IV})^{*}$ pair needs to be calculated. Doing this using only the Gibbs energy differences of the transition metal species involved has proven challenging in protic environments such as water. ${ }^{58}$ However, we provide a novel descriptor for the $\mathrm{p} K_{a}$, created by reproducing hypothetical Pourbaix diagrams and described in Supplementary Note 2. This is only possible if the OER descriptors are sampled at the correct oxidation states, leading to the ability to assess the resting state of the catalyst prior to the formation of the high-valent $\mathrm{M}(\mathrm{V})-\mathrm{O}$ species. Filtering on the basis of the $\mathrm{p} K_{a}$ leads to 3 promising Cr-based catalysts, highlighted within the red rectangle in Figure 6.
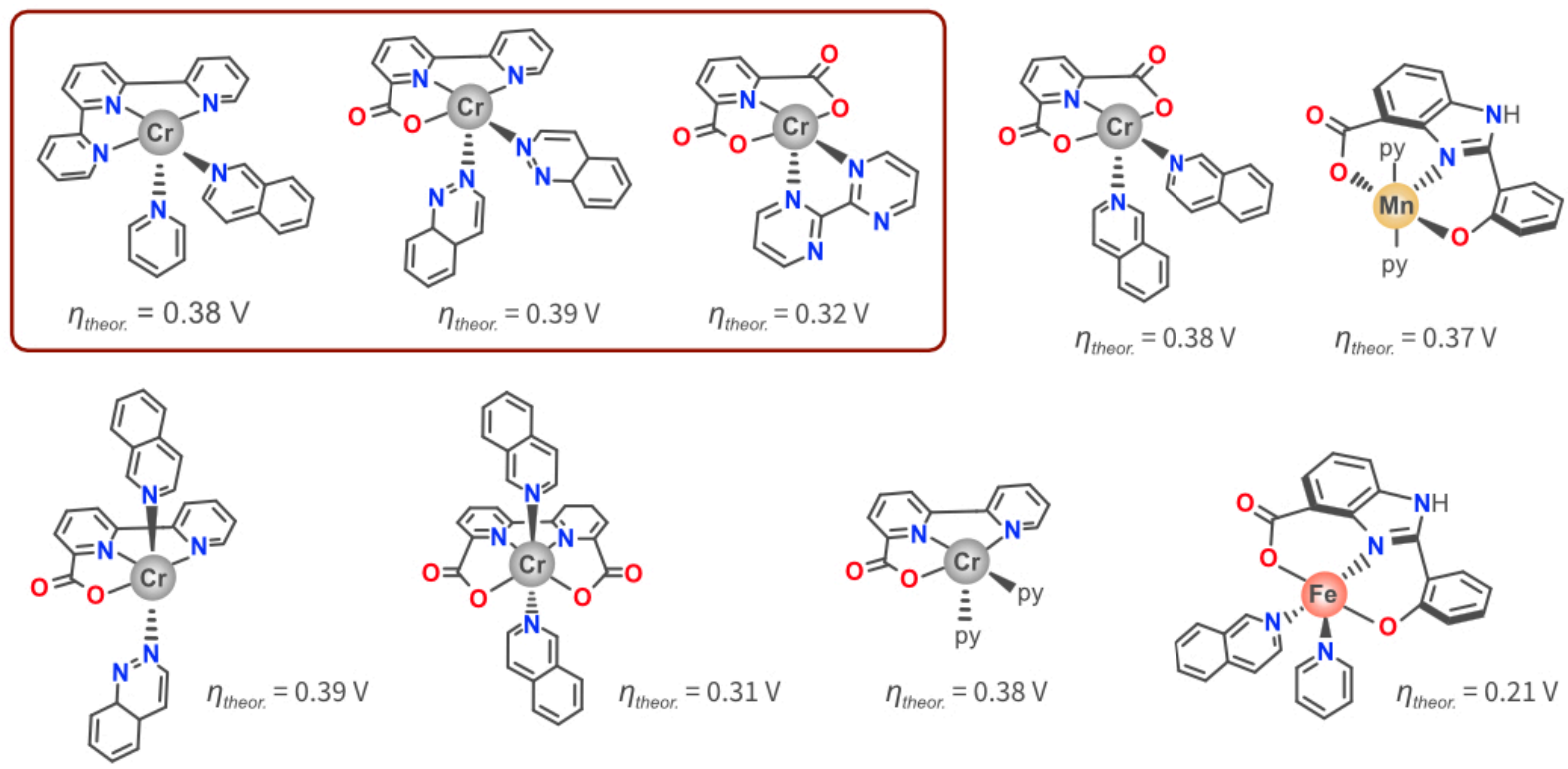

Figure 6. Catalysts found from the computational screening with overpotentials less than 0.4 $\mathrm{V}$, which have $\Delta G_{O(V)^{*}}<\Delta G_{H O O(I I I)^{*}}$ and $\Delta G_{H O O(I I I)^{*}}-\Delta G_{O(V)^{*}}<1 \mathrm{eV}$. The Cr catalysts highlighted in the red box are most promising having been further filtered on the basis that these catalysts can perform the extra oxidation at low $\mathrm{pH}$. The theoretical overpotential for 
each catalyst at $\mathrm{pH}=0$ is provided alongside each catalyst. The monodentate pyridine ligand is represented as py.

Each of the tailored catalysts shown in Figure 6 is based on a ligand which has been previously seen in molecular Ru OER catalysts. ${ }^{32}$ Assuming these catalysts can be made, experimental investigation will lead either to a refinement of our understanding of OER by rebutting our model, in the worst case, or novel low-cost catalysts with high activity in the best case. The primary issue foreseen for these catalysts would be the APT, and it must be noted that low overpotential does not guarantee a panacea, as kinetic limitations can present themselves by inhibiting $\mathrm{O}-\mathrm{O}$ bond formation. Engineering ligand functionalizations near the active site to address this has shown encouraging results in Ru catalysts, with carboxylate and phosphonate ligands enabling an enhancement of the WNA by orders of magnitude. ${ }^{59,60}$

In summary, this study presents the first automated approach to rational computational design of molecular OER catalysts based on earth-abundant elements. Through the application of key design principles and the fastidious choice of ligand scaffolds, we have solidified methodindependent scaling relations and provided fundamental insights into molecular water oxidation catalysts and the OER generally. We then proposed a link between deviations from our scaling relation in nickel catalysts to recent experimental OER observations. Further screening led us to develop metal-specific scaling relations between $\mathrm{O}^{*}$ and $\mathrm{HO}^{*}$ intermediates that did not exhibit typical behavior in expected slope, which we justified on the basis of a straightforward spin-based heuristic. As a result of the computational screening, we identified 9 low-overpotential, earth-abundant catalysts with theoretical overpotentials below $400 \mathrm{mV}$. We further filtered these catalysts based on their ability to evolve oxygen by the assumed mechanism in acid, leading to 3 promising Cr-based catalysts. Our data also has the potential to be used as the springboard for the machine learning-driven discovery of OER catalysts, 
further boosted by the scaling relations presented in Figures 3 and $5 \mathrm{~d}$. If the full potential of such ML models were realized they would help to avoid explorations of the chemical landscape that are not fruitful, while allowing researchers to trawl this space more effectively. The set of ligands used for this study were chosen due to their presence in other oxygen evolving complexes, therefore, it is inherently restricted by what mononuclear OER catalysts already exist. This could be overcome when generative models, which have been used to faithfully reproduce organic molecules,${ }^{61}$ are applied to transition metal complexes. Then, machine learning approaches will become even more powerful, with the ability to amplify the effects of high-speed predictions while testing catalysts over a much broader region of chemical space. Future work will focus on studying the stability of these complexes against ligand oxidation or replacement.

Going beyond trial-and-error catalyst discovery for renewable energy technologies will require different approaches. This work emphasizes that large-scale computational analyses are one such approach in homogeneous OER catalysis.

\section{Computational Methods}

DFT calculations were performed at the TPSSh, M06-L and B3LYP level using the Gaussian09 software, while BEEF-vdW calculations were performed using the Vienna Ab Initio Simulation Package (VASP) code, version 5.4.4. ${ }^{62-65}$ The results of the M06-L, B3LYP and BEEF-vdW calculations are presented in Supplementary Figure 2.

To describe the Ru, Mn, Fe, Cr, Co and Ni metals using Gaussian09, the Lanl2dz effective core potential was used along with $f$-polarization functions with exponents $1.235,2.195,2.462$, 1.941, 2.78 and 3.13, respectively ${ }^{66}$ The more electronegative $\mathrm{O}, \mathrm{N}, \mathrm{F}$, and $\mathrm{Cl}$ atoms were described using the $6-31+\mathrm{G}(\mathrm{d})$ basis set, while the $6-31 \mathrm{~g}(\mathrm{~d}, \mathrm{p})$ basis set was employed for $\mathrm{C}$ and $\mathrm{H}$ atoms. Molecular structures were optimized in water using the TPSSh functional and 
the implicit SMD solvation model $(\varepsilon=78.3553) .{ }^{67} \mathrm{We}$ confirmed that TPSSh geometries are satisfactory in comparison to experimental bond lengths and ensured they correspond to minima on the potential energy surface via vibrational frequency calculations, while a recent investigation found this functional to provide the lowest error in reproducing experimental redox potentials. ${ }^{10}$ These geometries were used to generate the data with the B3LYP and M06L functionals via single point calculations. Gibbs energy corrections obtained from the TPSSh frequency calculations were then added to the potential energies computed with B3LYP and M06-L. Gibbs energies were calculated at the temperature of $298.15 \mathrm{~K}$ and pressure of $1 \mathrm{~atm}$, except for the isolated $\mathrm{H}_{2} \mathrm{O}$ molecule that was computed at the temperature and pressure at which both the liquid and gas phases are in equilibrium, i.e. $300 \mathrm{~K}$ and $0.035 \mathrm{~atm}$. Relative Gibbs energies are referenced to $\mathrm{H}_{2} \mathrm{O}$ and $\mathrm{H}_{2}$ in solution, to avoid introducing the error associated with the modelling of $\mathrm{O}_{2}$ with DFT methods, and the global reaction Gibbs energy was fixed to the experimental value of $4.92 \mathrm{eV}$. To ensure sound geometries, we inspect any intermediate if atoms coordinated to the metal change, or if a bond distance changes by $20 \%$ or more. Grimme D3 dispersion corrections ${ }^{68}$ were added via single point calculations at the optimized geometries, except for the results of M06-L calculations, as this Minnesota functional was partly fit to noncovalent interactions.

BEEF-vdW calculations in VASP-5.4.4 were performed using projector-augmented wave pseudopotentials ${ }^{69}$ and plane waves with a kinetic energy cutoff of $500 \mathrm{eV}$. Molecular catalysts were modelled in a unit cell with a vacuum spacing of at least $15 \AA$ in each direction and at the $\Gamma$-point. A self-consistent evaluation of the wavefunction was stopped once the electronic energy changed by less than $1 \times 10^{-6} \mathrm{eV}$, while geometry optimization completed when all forces on atoms were within $0.02 \mathrm{eV} / \AA ̊$. Gibbs energy corrections - using the same conditions outlined in the previous paragraph - of $0.31 \mathrm{eV}$ and $0.4 \mathrm{eV}$ were added to $\Delta E_{H O^{*}}$ and $\Delta E_{H O O^{*}}$ to arrive at $\Delta G_{H O^{*}}$ and $\Delta G_{H O O^{*}}$, respectively. These corrections were obtained by taking the 
average of the Gibbs corrections of a Ru and Fe catalyst, which only varied by at most 0.02 $\mathrm{eV}$. These values were derived for individual adsorbates using the ideal gas approximation, deriving the zero-point energy, translational, rotational, vibrational and electronic inputs to the heat capacity at fixed pressure at $298.15 \mathrm{~K}, 1$ atm along with the entropic contributions via the Thermochemistry module provided by the Atomic Simulation Environment. ${ }^{70}$

The set of ligands seen in Figure 2 were chosen as they had seen application in experimentally realized catalysts. The charges of these ligands were pre-assigned and used to define the total charge of the catalyst. The spin state of metals in each reaction intermediate was determined by investigating which multiplicity was the most stable for a representative set of molecules. In particular, if for every metal in a certain oxidation state a specific multiplicity was always lower in energy by $0.3 \mathrm{eV}$, that spin state was assigned throughout for all calculations. Following this criterion, all $\mathrm{Mn}$ oxidation states, $\mathrm{Fe}(\mathrm{II}), \mathrm{Co}(\mathrm{II}), \mathrm{Cr}(\mathrm{II})$ were assigned to be highspin, while all $\mathrm{Ru}$ oxidation states, $\mathrm{Co}(\mathrm{III}), \mathrm{Ni}(\mathrm{II})$, were assigned low-spin. For Fe(III) complexes, both high and low-spin structures were modelled as no prevalent spin-state was determined. The results of this investigation are shown in Supplementary Table S2. The spinstates exhibiting the lowest energy were subsequently modelled using the M06-L and B3LYP exchange-correlation functionals, while the low-spin state was modelled regardless of the lowest energy spin state from TPSSh as GGA functionals are known to favor low-spin states. ${ }^{71}$

\section{Acknowledgments}

Authors gratefully thank Trinity College Dublin for financial support. Particularly, M. J. C. and M. G.-M. acknowledge funding from the Provost's PhD Project Awards, generously funded through alumni donations and Trinity's Commercial Revenue Unit. M. J. C. also acknowledges support from the Irish Research Council under Grant No. GOIPG/2019/2367. The DJEI/DES/SFI/HEA Irish Centre for High-End Computing (ICHEC) is acknowledged for the 
generous provision of computational facilities and support. Authors also thank Mr. Eric MatesTorres for providing assistance with the graphical design.

\section{Author Contributions}

M. G.-M. designed and supervised the work. M. J. C carried out all the computational studies.

M. J. C. and M. G.-M. analyzed and discussed all the data and cowrote the manuscript.

\section{Competing Interests}

The authors declare no competing interests.

\section{Materials \& Correspondence}

Correspondence should be addressed to: garciamm@tcd.ie

\section{Supplementary Information}

Additional data underlying this work is available online.

\section{Data Availability}

All calculated structures are to be found at an ioChem-BD repository currently under embargo.

\section{References}

1. Seh, Z. W. et al. Combining theory and experiment in electrocatalysis: Insights into materials design. Science 355, eaad4998 (2017).

2. Ramachandran, R. An overview of industrial uses of hydrogen. Int. J. Hydrogen Energy 23, 593-598 (1998).

3. Acar, C. \& Dincer, I. Comparative assessment of hydrogen production methods from renewable and non-renewable sources. Int. J. Hydrogen Energy 39, 1-12 (2014). 
4. Gao, D., Arán-Ais, R. M., Jeon, H. S. \& Roldan Cuenya, B. Rational catalyst and electrolyte design for $\mathrm{CO} 2$ electroreduction towards multicarbon products. Nat. Catal. 2, 198-210 (2019).

5. Nitopi, S. et al. Progress and Perspectives of Electrochemical $\mathrm{CO}_{2}$ Reduction on Copper in Aqueous Electrolyte. Chem. Rev. 119, 7610-7672 (2019).

6. Soriano-López, J., Schmitt, W. \& García-Melchor, M. Computational modelling of water oxidation catalysts. Curr. Opin. Electrochem. 7, 22-30 (2018).

7. Kurth, Stefan, Perdew, John P., \& Blaha, Peter. Molecular and Solid-State Tests of Density Functional Approximations: LSD, GGAs, and Meta-GGAs. Int. J. Quantum Chem. 75, 889-909 (1999).

8. Nørskov, J. K. et al. Origin of the Overpotential for Oxygen Reduction at a Fuel-Cell Cathode. J. Phys. Chem. B 108, 17886-17892 (2004).

9. Man, I. C. et al. Universality in Oxygen Evolution Electrocatalysis on Oxide Surfaces. ChemCatChem, 3, 1159-1165 (2011).

10. Craig, M. J. et al. Universal scaling relations for the rational design of molecular water oxidation catalysts with near-zero overpotential. Nat. Commun. 10, 4993 (2019).

11. Busch, M., Fabrizio, A., Luber, S., Hutter, J. \& Corminboeuf, C. Exploring the Limitation of Molecular Water Oxidation Catalysts. J. Phys. Chem. C 122, 12404-12412 (2018).

12. Baran, J. D., Grönbeck, H. \& Hellman, A. Analysis of Porphyrines as Catalysts for Electrochemical Reduction of $\mathrm{O}_{2}$ and Oxidation of $\mathrm{H}_{2} \mathrm{O}$. J. Am. Chem. Soc. 136, 1320 $1326(2014)$.

13. Calle-Vallejo, F., Martínez, J. I., García-Lastra, J. M., Abad, E. \& Koper, M. T. M. Oxygen reduction and evolution at single-metal active sites: Comparison between functionalized graphitic materials and protoporphyrins. Surf. Sci. 607, 47-53 (2013). 
14. Craig, M. J., Barda-Chatain, R. \& García-Melchor, M. Fundamental insights and rational design of low-cost polyoxometalates for the oxygen evolution reaction. J. Catal.393,202$206(2021)$.

15. Shamsipur, M., Taherpour, A. (Arman), Sharghi, H., Lippolis, V. \& Pashabadi, A. A lowoverpotential nature-inspired molecular chromium water oxidation catalyst. Electrochim. Acta 265, 316-325 (2018).

16. Fillol, J. L. et al. Efficient water oxidation catalysts based on readily available iron coordination complexes. Nat. Chem. 3, 807-813 (2011).

17. Codolà, Z. et al. Electronic Effects on Single-Site Iron Catalysts for Water Oxidation. Chem. Eur. J. 19, 8042-8047 (2013).

18. Ellis, W. C., McDaniel, N. D., Bernhard, S. \& Collins, T. J. Fast Water Oxidation Using Iron. J. Am. Chem. Soc. 132, 10990-10991 (2010).

19. Young, K. J., Takase, M. K. \& Brudvig, G. W. An Anionic N-Donor Ligand Promotes Manganese-Catalyzed Water Oxidation. Inorg. Chem. 52, 7615-7622 (2013).

20. Wasylenko, D. J., Ganesamoorthy, C., Borau-Garcia, J. \& Berlinguette, C. P. Electrochemical evidence for catalytic water oxidation mediated by a high-valent cobalt complex. Chem. Commun. 47, 4249 (2011).

21. Pizzolato, E. et al. Light driven water oxidation by a single site cobalt salophen catalyst. Chem. Commun. 49, 9941 (2013).

22. Fu, S. et al. A mononuclear cobalt complex with an organic ligand acting as a precatalyst for efficient visible light-driven water oxidation. Chem. Commun. 50, 2167-2169 (2014).

23. Wang, D. \& Groves, J. T. Efficient water oxidation catalyzed by homogeneous cationic cobalt porphyrins with critical roles for the buffer base. Proc. Natl. Acad. Sci. USA 110, 15579-15584 (2013). 
24. Han, Y., Wu, Y., Lai, W. \& Cao, R. Electrocatalytic Water Oxidation by a Water-Soluble Nickel Porphyrin Complex at Neutral pH with Low Overpotential. Inorg. Chem.54, 5604$5613(2015)$.

25. Coggins, M. K., Zhang, M.-T., Chen, Z., Song, N. \& Meyer, T. J. Single-Site Copper(II) Water Oxidation Electrocatalysis: Rate Enhancements with $\mathrm{HPO}_{4}{ }^{2-}$ as a Proton Acceptor at pH 8. Angew. Chem. 53, 12226-12230 (2014).

26. Barnett, S. M., Goldberg, K. I. \& Mayer, J. M. A soluble copper-bipyridine wateroxidation electrocatalyst. Nat. Chem. 4, 498-502 (2012).

27. Duan, L. et al. A molecular ruthenium catalyst with water-oxidation activity comparable to that of photosystem II. Nat. Chem. 4, 418-423 (2012).

28. Duan, L., Araujo, C. M., Ahlquist, M. S. G. \& Sun, L. Highly efficient and robust molecular ruthenium catalysts for water oxidation. Proc. Natl. Acad.Sci. USA 109, 1558415588 (2012).

29. Matheu, R. et al. Intramolecular Proton Transfer Boosts Water Oxidation Catalyzed by a Ru Complex. J. Am. Chem. Soc. 137, 10786-10795 (2015).

30. Craig, M. J. \& García-Melchor, M. Faster hydrogen production in alkaline media. Nat. Catal. 3, 967-968 (2020).

31. Ioannidis, E. I., Gani, T. Z. H. \& Kulik, H. J. molSimplify: A toolkit for automating discovery in inorganic chemistry. J. Comp. Chem. 37, 2106-2117 (2016).

32. Kärkäs, M. D., Verho, O., Johnston, E. V. \& Åkermark, B. Artificial Photosynthesis: Molecular Systems for Catalytic Water Oxidation. Chem. Rev. 114, 11863-12001 (2014).

33. Govindarajan, N., Tiwari, A., Ensing, B. \& Meijer, E. J. Impact of the Ligand Flexibility and Solvent on the O-O Bond Formation Step in a Highly Active Ruthenium Water Oxidation Catalyst. Inorg. Chem. 57, 13063-13066 (2018). 
34. Staroverov, V. N., Scuseria, G. E., Tao, J. \& Perdew, J. P. Comparative assessment of a new nonempirical density functional: Molecules and hydrogen-bonded complexes. $J$. Chem. Phys. 119, 12129-12137 (2003).

35. Becke, A. D. Density-functional thermochemistry. III. The role of exact exchange. $J$. Chem. Phys. 98, 5648-5652 (1993).

36. Zhao, Y. \& Truhlar, D. G. A new local density functional for main-group thermochemistry, transition metal bonding, thermochemical kinetics, and noncovalent interactions. J. Chem. Phys. 125, 194101 (2006).

37. Wellendorff, J. et al. Density functionals for surface science: Exchange-correlation model development with Bayesian error estimation. Phys. Rev. B 85, 235149 (2012).

38. Seitz, L. C. et al. A highly active and stable $\mathrm{IrO}_{x} / \mathrm{SrIrO}_{3}$ catalyst for the oxygen evolution reaction. Science 353, 1011-1014 (2016).

39. Christensen, R., Hansen, H. A., Dickens, C. F., Nørskov, J. K. \& Vegge, T. Functional Independent Scaling Relation for ORR/OER Catalysts. J. Phys. Chem. C 120, 2491024916 (2016).

40. Garcés-Pineda, F. A., Blasco-Ahicart, M., Nieto-Castro, D., López, N. \& Galán-Mascarós, J. R. Direct magnetic enhancement of electrocatalytic water oxidation in alkaline media. Nat. Energy 4, 519-525 (2019).

41. Pérez-Ramírez, J. \& López, N. Strategies to break linear scaling relationships. Nat. Catal. 2, 971-976 (2019).

42. Matheu, R., Ertem, M. Z., Gimbert-Suriñach, C., Sala, X. \& Llobet, A. Seven Coordinated Molecular Ruthenium-Water Oxidation Catalysts: A Coordination Chemistry Journey: Focus Review. Chem. Rev. 119, 3453-3471 (2019).

43. Gracia, J. Spin dependent interactions catalyse the oxygen electrochemistry. Phys. Chem. Chem. Phys. 19, 20451-20456 (2017). 
44. Jiao, Y., Sharpe, R., Lim, T., Niemantsverdriet, J. W. H. \& Gracia, J. Photosystem II Acts as a Spin-Controlled Electron Gate during Oxygen Formation and Evolution. J. Am. Chem. Soc. 139, 16604-16608 (2017).

45. Gracia, J., Sharpe, R. \& Munarriz, J.Principles determining the activity of magnetic oxides for electron transfer reactions. J. Catal. 361, 331-338 (2018).

46. Zhang, B. et al. Homogeneously dispersed multimetal oxygen-evolving catalysts. Science 352, 333-337 (2016).

47. $\mathrm{Ng}, \mathrm{J} . \mathrm{W}$. D. et al. Gold-supported cerium-doped NiOx catalysts for water oxidation. Nat. Energy 1, 16053 (2016).

48. Huynh, M. H. V. \& Meyer, T. J. Proton-Coupled Electron Transfer. Chem. Rev. 107, 5004-5064 (2007).

49. García-Melchor, M., Vilella, L., López, N. \& Vojvodic, A. Computationally Probing the Performance of Hybrid, Heterogeneous, and Homogeneous Iridium-Based Catalysts for Water Oxidation. ChemCatChem 8, 1792-1798 (2016).

50. Coggins, M. K., Zhang, M.-T., Vannucci, A. K., Dares, C. J. \& Meyer, T. J. Electrocatalytic Water Oxidation by a Monomeric Amidate-Ligated Fe(III)-Aqua Complex. J. Am. Chem. Soc. 136, 5531-5534 (2014).

51. Craig, M. \& Garcia-Melchor, M. Discerning Activity and Inactivity in Earth-Abundant Molecular Water Oxidation Catalysts. CshemCatChem 12, 4775-4779 (2020).

52. Hammer, B. \& Norskov, J. K. Why gold is the noblest of all the metals. Nature 376, 238$240(1995)$.

53. Gunasooriya, G. T. K. K. \& Nørskov, J. K. Analysis of Acid-Stable and Active Oxides for the Oxygen Evolution Reaction. ACS Energy Lett. 5, 3778-3787 (2020).

54. Abild-Pedersen, F. et al. Scaling Properties of Adsorption Energies for HydrogenContaining Molecules on Transition-Metal Surfaces. Physical Review Letters 99, (2007). 
55. Wiberg, K. B. Application of the pople-santry-segal CNDO method to the cyclopropylcarbinyl and cyclobutyl cation and to bicyclobutane. Tetrahedron 24, 10831096 (1968).

56. Bhattacharjee, S., Waghmare, U. V. \& Lee, S.-C. An improved d-band model of the catalytic activity of magnetic transition metal surfaces. Sci Rep 6, 35916 (2016).

57. Wasylenko, D. J., Palmer, R. D., Schott, E. \& Berlinguette, C. P. Interrogation of electrocatalytic water oxidation mediated by a cobalt complex. Chem. Commun. 48, 2107 (2012).

58. Sinha, V., Laan, J. J. \& Pidko, E. A. Accurate and rapid prediction of $\mathrm{p} K_{\mathrm{a}}$ of transition metal complexes: semiempirical quantum chemistry with a data-augmented approach. Phys. Chem. Chem. Phys. 23, 2557-2567 (2021).

59. Vereshchuk, N. et al. Second Coordination Sphere Effects in an Evolved Ru Complex Based on Highly Adaptable Ligand Results in Rapid Water Oxidation Catalysis. J. Am. Chem. Soc. 142, 5068-5077 (2020).

60. Shaffer, D. W., Xie, Y., Szalda, D. J. \& Concepcion, J. J. Lability and Basicity of Bipyridine-Carboxylate-Phosphonate Ligand Accelerate Single-Site Water Oxidation by Ruthenium-Based Molecular Catalysts. J. Am. Chem. Soc. 139, 15347-15355 (2017).

61. Sanchez-Lengeling, B. \& Aspuru-Guzik, A. Inverse molecular design using machine learning: Generative models for matter engineering. Science 361, 360-365 (2018).

62. Kresse, G. \& Hafner, J. Ab initio molecular dynamics for liquid metals. Phys. Rev. B 47, $558-561(1993)$.

63. Kresse, G. \& Hafner, J. Ab initio molecular-dynamics simulation of the liquid-metalamorphous-semiconductor transition in germanium. Phys. Rev. B 49, 14251-14269 (1994). 
64. Kresse, G. \& Furthmüller, J. Efficient iterative schemes for ab initio total-energy calculations using a plane-wave basis set. Phys. Rev. B 54, 11169-11186 (1996).

65. Kresse, G. \& Furthmüller, J. Efficiency of ab-initio total energy calculations for metals and semiconductors using a plane-wave basis set. Comput. Mater. Sci. 6, 15-50 (1996).

66. Ehlers, A. W. et al. A set of f-polarization functions for pseudo-potential basis sets of the transition metals Sc-Cu, Y-Ag and La-Au. Chem. Phys. Lett. 208, 111-114 (1993).

67. Marenich, A. V., Cramer, C. J. \& Truhlar, D. G. Universal Solvation Model Based on Solute Electron Density and on a Continuum Model of the Solvent Defined by the Bulk Dielectric Constant and Atomic Surface Tensions. J. Phys. Chem. B 113, 6378-6396 (2009).

68. Grimme, S., Antony, J., Ehrlich, S. \& Krieg, H. A consistent and accurate ab initio parametrization of density functional dispersion correction (DFT-D) for the 94 elements H-Pu. J. Chem. Phys. 132, 154104 (2010).

69. Blöchl, P. E. Projector augmented-wave method. Phys. Rev. B 50, 17953-17979 (1994).

70. Hjorth Larsen, A. et al. The atomic simulation environment - a Python library for working with atoms. J. Phys. Condens. Matter 29, 273002 (2017).

71. Ioannidis, E. I. \& Kulik, H. J. Ligand-Field-Dependent Behavior of Meta-GGA Exchange in Transition-Metal Complex Spin-State Ordering. J. Phys. Chem. A 121, 874-884 (2017). 